\title{
FURTHER CONSIDERATION OF SOME OF THE PROBLEMS OF TREATMENT OF PULPLESS TEETH, INCLUDING REMARKS ON RECENT CRITICISMS BY OTTOLENGUI ${ }^{2}$
}

\author{
M. L. RHEIN \\ Nero York City \\ CONTENTS
}

I. Introduction ....................................... 327

II. Development of a satisfactory procedure for the complete removal of pulp

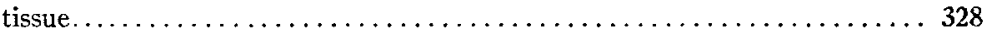

III. On the necessity for passing the pulp-removing material through the foramen. 331

IV. The removal of all diseased periapical tissue is essential; the utility of electrolytic treatment for this purpose $\ldots \ldots \ldots \ldots \ldots \ldots \ldots \ldots \ldots \ldots \ldots . \ldots \ldots \ldots \ldots$

V. A proportion of failures is inevitable in every surgical procedure ......... 337

VI. Remarks on some of Ottolengui's recent criticisms ................. 338

VII. The importance of encapsulation of all the foraminal openings......... 338

VIII. Discussion of the illustrations (figs. 1-24); and further comment on criticisms

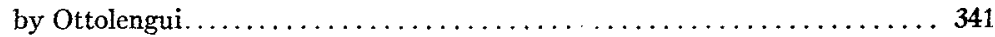

IX. Summary of general views................................ 353

\section{INTRODUCTION}

During the period of my last year in a medical school, I devoted considerable attention to dental technics in the office of my father. The subject of pulp removal and root-canal fillings was a matter of much controversy between us. I urged him to save pulpless teeth, but he refused to leave any such teeth in the mouths of his patients. During the last year of my course in medicine, my father repeatedly called my attention to the ill health and discomforts of patients with root fillings in pulpless teeth, and their improved health after the removal of such teeth. The germ theory had just been born; and at that time (1880) I commenced experimental work on the possibility of so operating on pulpless teeth that they would not be a menace to health.

1 The main portion of the text was read before the Pathodontia Section of the First District Dental Society of the State of New York, at the New York Academy of Medicine, April 26, 1921. 
At the period of these early experiences, medicine had just passed through "the Listerian period." During an operation by the Listerian surgical technique, a number of assistants sprayed the patient, the operator, the field of operation and all his immediate environment, with solutions of phenol, in order to prevent infection. The commencement of our present-day method of aseptic surgery had just been heralded. The celebrated gynecologist, Sir Lawrence Tait, of Birmingham, England, astonished the surgical world with reports of the large percentage of recoveries in his cases of ovariotomy. As far as Tait was concerned, carbolic acid was a thing of the past; he boiled all his instruments, and used the most rigid care to obtain absolute cleanliness both in regard to the field of operation, and to his own hands and person and those of his assistants. After ceasing to use a knife or an instrument taken from the pan of boiling water, it was never re-used during the operation. In this way was born aseptic surgery of the present day. Its value was deeply impressed upon me because I was delegated by my preceptor; Dr. A. Vanderveer, of Albany, to welcome Tait to the United States, and it became my pleasant duty to assist him in many of the operations he performed here and in Albany.

My brief association with Sir Lawrence Tait gave me the impulse to attempt to handle root canals on this basis. From that time on my instruments were boiled, and creosote and carbolic acid were removed from my armamentarium, much to the pleasure of my few but sensitive patients. It must be remembered that creosote had been the foundation stone upon which all root-canal technic was based. The more foul smelling the creosote, the more favor it met from our best operators.

\section{DEVELOPMENT OF A SATISFACTORY PROCEDURE FOR THE COMPLETE REMOVAL OF PULP TISSUE}

From the beginning it was my object mechanically to remove all pulp tissue, place the canal in an aseptic condition, and then hermetically seal it with solid base-plate gutta-percha. In the early eighties, I introduced the solution of sublimate in Marchand's hydrogen peroxide, in order to obtain a non-septic root canal. By irrigating the canal with this solution, the free oxygen caused the loose particles 
of debris to come to the surface; and I depended on the minute deposits of sublimate to keep the canal free from germs.

Shortly after the beginning of my work in this relation, William H. Atkinson and I experimented on the amputation of vital pulps. For some little time we were encouraged by seeming success, but the crop of abscesses that followed soon undeceived us. About that time (1885), Herbst, of Bremen, Germany, introduced the practice of pulp mummification, by removing the pulp from the crown only and by sealing in the canal a small amount of "cobalt."

I saw Herbst give a clinic with cobalt and my pathologic sense was outraged by it. I have never ceased to object to this form of therapy. During this period I was closely associated with William H. Atkinson, to whose farseeing pathologic sense, I feel, we have been deeply indebted. The roentgenogram had not yet been invented, and our clinics on root-canal technic, with their tedious time-taking service, found little favor with the profession, who were being more and more led astray by the exponents of pulp mummification. When the great Miller, of Berlin, already famous as a bacteriologist, wrote a paper, for Dental Cosmos, advocating mummification and publishing his formula for this purpose, he weakened the faith of many of our disciples.

This was the most trying period of my research in this field, because I was discouraged by the percentage of failures that I could not fail to recognize, necessitating extractions, which did not increase my popularity with my patients. It was about this period that Callahan introduced a solution of sulphuric acid for the purpose of chemically enlarging semi-calcified root canals. All the other powerful acids alone and in combination were at once experimented with. While we found that the sulphuric acid was of the greatest value in obtaining better results, it fell short of solving the problem of removing every vestige of pulp tissue. It failed utterly to destroy the inaccessible pulp tissue that was free from calcification. in fact, it only brought more adherence to the practice of mummification.

Greatly discouraged at being so near to, yet so far from, the goal of removing every particle of pulp tissue, I attended the First International Dental Congress, held in Chicago in 1893, where I was destined to find the chemical agents which have enabled us to over- 
come that greatest of obstacles-the removal of pulp tissue from inaccessible canals and ramifying branches.

During the sessions of the Congress, Emil Schreier, of Vienna, gave continuous clinics with his mixture of sodium and potassium (kalium-natrium). It was the most popular clinic at that great meeting of dentists. He introduced, and sealed in the root canals, a mass of his amalgam compound in the same way in which all the mummifying preparations were being used. It was supposed to be the latest formula for pulp mummification. He had invented a method of combining these two metals, sodium and potassium.

I watched Schreier's first clinic with a feeling of skepticism, but was at once fascinated by the same thing that attracted everyone. When this amalgam-like mixture came in contact with pulp tissue, there was generally an explosion, frequently a flame, much gas, but nothing remained of that particular portion of pulp tissue. Again and again $\mathrm{I}$ returned to his clinic to watch this destruction of pulp tissue, and realized that the agent had been finally invented by a dentist which could remove every vestige of pulp tissue contained within the root canals. Schreier visited me on his way through New York, and then I demonstrated to him in my office that its real value consisted in his capability of destroying every vestige of organic substance.

Sodium-potassium should be introduced in the smallest quantity, in order to destroy a limited amount of tissue at a time. Used in large masses there is always danger of destroying organic cement substance in the dentine itself. The destructive power of this agent is very much increased by the intense heat resulting from the chemical union of the water in the organic substance with the sodium-potassium. There is an entire absence of escharotic action such as results when sulphuric acid is used under similar conditions.

After its escharotic action has been accomplished, sulphuric acid leaves an inorganic precipitate, which cannot be absorbed. While this result is harmless in the canals, it is not a satisfying reaction for the periapical region. 


\section{ON THE NECESSITY FOR PASSING THE PULP-REMOVING MATERIAL THROUGH THE FORAMEN}

To obtain the maximum benefit from the use of sodium-potassium, the material should be passed through the foramen. No one should hesitate to use it in this way, even in cases of removal of vital pulps. The ultimate junction of pulp tissue, at the foramen, with periapical tissue, has often been spoken of as the physiologic encapsulation of the root end. My clinical records show many cases where this small amount of living tissue soon died and became the initial factor in a focal infection. The fact that this small amount of pulp tissue may retain its vitality for variable periods of time, in some cases, does not warrant its retention in any instance, when we consider that many cases of infection arise solely from this reaction to lack of operative thoroughness. There are, of course, exceptions to this rule of practice. W. Clyde Davis has recently called our attention to a series of pulpless teeth where new cementum has sealed the foraminal entrances and extends some distance up the canal. These results undoubtedly are found in only a small proportion of teeth. If the diagnosis of this condition can be made, there ought to be no necessity for attempting to go further than the root filling of cementum, as this leaves an ideal encapsulation if the new cementum hermetically seals the foramina. This diagnosis is not easily made, but it will undoubtedly be frequently given as an excuse for imperfect and slovenly work. Incidentally, it might be stated that the cases reported by Davis afford an additional argument against the absurdity of regarding a pulpless tooth as necessarily a dead tooth. ${ }^{2}$

About eighteen months ago, in collaboration with Drs. Gies and Bastian, we injected trypan blue into two dogs. These injections

2 Since the above was written, Davis has withdrawn his statement on the possibility that cementum can fill the dental foramen, and assumes that, in the cases he has had under observation, calcification is in progress, which he calls "osteoid." This simply expresses an observation, on his part, of the ordinary calcific degeneration that occurs in so large a percentage of root canals. Careful observation on my part, covering a period of over forty years, has shown that this calcific matter is intermingled here and there with organic layers, which easily become seats of infection. It also demonstrates the lack of dependence that can be placed upon such hasty and careless observations as those of Davis. His work in this connection is simply another effort in the field of embalming (Egyptian dentistry!) No words of warning can be too strong against the profession's giving serious consideration to doctrines such as these. 
were repeated at intervals until we established a well marked blue coloration of each dog. The pulp of two of the incisors in each of these dogs had previously been removed and good gutta-percha root fillings inserted. At a later period, after these dogs had been chloroformed, the jaws were very carefully examined. It was observed that the trypan blue had found its way into all of the tissues, hard and soft, including the bones and the teeth. Individual teeth were removed from the jaw, split, and examined carefully. The periodontal membrane in each case was found to be thoroughly impregnated by the pigment, and the teeth themselves assumed a distinctly bluish tint. As our research in this direction is to continue, I prefer to postpone discussion of this comparison. One finding was impressive. Although the periodontal membrane around the apex was completely discolored by the trypan blue, none of this pigment passed through the foramen into the canal. The pink gutta-percha root filling failed to show a trace of discoloration. This must be considered good evidence that base-plate gutta-percha and chloroform will hermetically seal root canals.

In an investigation I reported to this Section over a year ago, the apical thirds of some teeth were covered with gutta-percha, chloroform and rosin; others, with chloroform and rosin; and others, with chloroform and gutta-percha. These specimens were tightly sealed, some in vials of distilled water and some in physiologic salt solution. Within thirty-six hours the water in the vials holding any mixture of rosin contained numerous organisms that continued rapidly to multiply. The water in the vials containing teeth covered with gutta-percha and chloroform remained unchanged during weeks of observation. I also called attention to the fact that rosin and chloroform fail to yield a perfect mixture. Their mechanical mixture is a mass filled with air bubbles, which tend to make the mixture exceedingly brittle when it finally hardens. This condition has accounted for the fact that a great many encapsulations have broken away from the end of the root, and it is very timely to have it understood that gutta-percha and rosin root-fillings should never be confounded with a correct base-plate gutta-percha root filling. The latter material never changes its physical characteristics after it has once been placed in position. All rosins contain numerous varieties 
of organisms and consequently the use of gutta-percha and rosin must be condemned.

The use of sodium-potassium, as above described, has been severely criticized by different men. This criticism has been mainly directed against its destruction of tissue in the periapical region. From a general dental standpoint, Grove and Grieves appear to have made out a very strong case against its use. Let us consider, for a moment, the bugaboo of periapical destruction caused by the use of sodiumpotassium. It destroys tissue, diseased and healthy, and its use can be appropriately compared to that of the surgeon's knife. Good surgery demands not only the removal of the diseased mass, but also that of surrounding healthy tissue. The surgeon does this fearlessly because of the recognized regenerative power of all tissue, unless infective forces are present. To criticize a surgeon for removing healthy tissue in the environment of a diseased mass would receive no serious attention in any surgical assemblage. In our therapy, sodium-potassium takes the place of the knife, a fact that should be a sufficient reply to criticism of this nature.

When a root canal is opened by utilizing the chemical action of sodium-potassium, by the very virtue of its action as it advances toward the foramen of the tooth, infection is automatically eliminated. Its own destructive action makes it impossible to force infected pulp through a foramen. Blocking the nerve trunk with novocain, as a local anesthetic, generally renders the use of sodium-potassium entirely painless, whenever this is found to be required.

Grieves is deserving of great praise for his excellent classification of pulpily and apically diseased teeth. There is no reason why this classification should not be generally accepted. His conclusions, however, as to treatment, are wholly at variance with my own views, which are based entirely on clinical data that can be best expressed as follows: All pulp tissue having been removed and periapical disease, if existent, eradicated, successful root-canal therapy is in the last analysis dependent on ideal root encapsulation. The basis of this claim rests on evidence from roentgenograms taken year after year after such encapsulation.

At the very commencement of my practice, I began to look to surgery for aid in removing all diseased periapical tissue, and soon 
was amputating root ends as a regular routine of practice. I presented this operation before the American Dental Association at Excelsior Springs in 1890 . Where scientific root-canal treatment has been used, there should be very little difficulty in surgical removal of infected tissue. The postoperative danger in these cases lurks in a possible osteoclasia, resulting in destruction of the root end.

In some recent research, Hopewell-Smith has shown that, besides the danger of osteoclasia, root ends are subject to caries. This undoubtedly shows the presence of bacteria deeply lodged in the alveolar structure. My clinical observation, for over forty years, long ago led me to conclude that presence of a great amount of infected tissue is the cause of operative failure in many cases. The many postmortems that have shown heart muscle to be thoroughly impregnated with streptococci suggest how these organisms may, in like manner, be thoroughly imbedded around the apical region of a pulpless tooth; this tissue being for the time the locus minoris resistentiae. On this account, the destruction or eradication of a limited amount of infected periapical tissue is insufficient, in a percentage of cases, to restore healthiness to teeth. The proper therapy must protect exposed cementum or dentin from such attacks. The use of sodium-potassium at once produced a large decrease in the number of cases that called for apicoecotomy.

During the last year, Levene, of the Rockefeller Institute, suggested sodium methylate as a substitute for sodium-potassium, with the hope that it would be less irritating. Chayes has been the first to use it in dental practice. Its use, in my hands, has been less effective than that of sodium-potassium. Unfortunately, in my experience, there has not been less irritation. In the cases where I have used it, there has been irritation to a very marked extent. With novocain at our service, pain can be very largely eliminated. The important factor is use of the compound that will most effectively ream our canals and obliterate the organic tissue. For this purpose sodiumpotassium appears to be the most resourceful compound at our command. 
IV. THE REMOVAL OF ALI DISEASED PERIAPICAL TISSUE IS ESSENTIAL; THE UTILITY OF ELECTROLYTIC TREATMENT FOR THIS PURPOSE

The second requisite in root-canal therapy is the removal of all diseased tissue situated in the periapical region. This undoubtedly is most easily and thoroughly accomplished by surgical procedure. Unless this includes, however, the clean amputation of the end of the root, there is grave danger of destroying the periodontal lining at the end of the root, which invariably means re-infection. The danger of root absorption resulting from osteoclasia and caries is present in all forms of surgical procedure. On this account it was very desirable to find other means for the obliteration of pathologic conditions in the periapical region.

The utility of the galvanic current for cataphoric anesthesia very soon led me to employ it for the purpose of electrolytic medication. Since 1894 I have been using the current for this purpose. From a clinical standpoint I was soon impressed by the wonderful therapeutic value of the current that carried any desired ion into the periapical region. The number of resultant cures, and of teeth that were maintained free from re-infection, was certainly impressive. While its use is very largely empirical, clinical successes, repeated again and again, cannot be overlooked, and it only remains for intensive investigation to afford an explanation of the manner in which the curative results are obtained. This form of ionic medication requires an unending amount of patience and perseverence on the part of the dentist. At the very outset, in order to make the application painless, considerable time must be taken in the most careful gradation of the current until the largest possible amperage passes through the tooth without discomfort to the patient. Many of our best operators have failed in the use of electrolytic medication because of failure to force a sufficient amount of ions through the foramen. At the beginning, in special cases, cultures were taken at intervals from the periapical region; and the fact was noted that, in a large number of cases, no growth was obtained when these cultures were taken immediately after electrolization. When, however, the patient returned in a week, new cultures generally again yielded growths. This persistent clinical fact led me to use a continual increase in the degree of ionization in a given case. 
Three years ago, in collaboration with Drs. Gies and Levy, I began a compilation of statistics on this form of treatment. Since then every one of my cases of pulpless teeth has been subjected to careful culturing. This paper is not intended to be even a partial report on that research. I feel, however, that I have data enough to justify certain conclusions on the subject of electrolytic medication, the benefit of which should not be longer kept from the profession. Up to date I have sent to the Columbia University laboratory over forty-five hundred tubes to be cultured. Two remarkable facts have been noted. First: given a well defined region of infection, cultures taken from this region, immediately after thorough application of electrolytic medication, show no growth in over 90 per cent of the cases, although, in over 60 per cent of these cases, a second culture, taken a week later, yields a growth of streptococcus. These facts led to long continued use of constantly increasing quantities of current, often extending over a period of months, before filling the root canals. In many of these cases where the treatment extended over weeks, the total milli-amperage of applied current frequently amounted to as much as eight to ten milli-ampere hours. All of these cases have been kept under careful roentgenographic observation. This, in turn, has brought to light the second fact: wherever ideal encapsulation was possible, it was followed by ideal regeneration.

In an article published by Grieves, in the April (1921) number of the Journal of the National Dental Association, he lays great stress on the importance of the quality of roentgenograms, and the danger of confounding pathologic conditions with alveolar regeneration. He is very skeptical about alveolar regeneration. While admitting that it does sometimes occur, he says: "The trabeculae and partitions of the adjacent normal bone to which these should be compared are missing." The pictures of regenerated bone that I shall have the pleasure of showing you display all the trabeculae and partitions. I desire especially to call your attention to the gradual improvement, from a histologic viewpoint, of these tissues as time goes on. As the encapsulation becomes a year older, the markings of the trabeculae and partitions of the regenerated tissue become better defined. I feel, further, that I cannot agree with his statement that "apical rarefaction is diagnostic of a dead apex." The destruction of the 
alveolar structure by no means implies necessary damage to the periodontal lining; and as long as this membrane remains intact, with a sufficient circulation, it is most inappropriate to speak of the end of the root as being dead. There is no doubt that in some cases there is necrosis of the apex of the root. I have no knowledge, however, of any such case where it was not accompanied by suppuration of a character that always attends necrosis of bone or tooth structure. Such teeth should not be retained; but, on the other hand, they should not be confounded with teeth on which the pericementum surrounding the roots has been entirely destroyed.

\section{A PROPORTION OF FAILURES IS INEVITABLE IN EVERY SURGICAL PROCEDURE}

A certain percentage of failures occur in these operations as they do in all operations on the living body. Whenever the roentgenogram shows a failure in regeneration of the alveolar structure, it stamps the operation as a failure, and recourse must then be had to apicoectomy. Failing there, extraction of the tooth and currettement of the process are imperative. I shall show you pictures of successful operations. I shall show you, also, some cases of failures. In these failures we are confronted by the fact that the patient generally has no discomfort. Patients generally fail to have a proper understanding of the desirability of immediate removal of the tooth, etc. It is always necessary to make the patient thoroughly familiar with the reasons for a failure and with the danger of retaining such a tooth. Again, it demands the highest order of moral courage to acknowledge your failure when you know that, if you kept silent. the patient would never be any wiser. By admitting failure you run the risk of losing your patient, who in some instances seems to be unable to appreciate the virtue of your attitude. The crime of withholding such information from your patient, however, is obvious. In every other field of surgery, a percentage of failures is freely acknowledged. The same allowance will be made for dental surgery, when dentists freely admit, as they should, that there is a percentage of unavoidable failures. 
VI. REMARKS ON SOME OF OTTOLENGUI'S RECENT CRITICISMS

The June (1922) number of Dental Items of Interest publishes my discussion of a paper by Ottolengui on this subject. During that discussion I displayed a few of the roentgenograms reproduced in this paper. Ottolengui has seen fit to criticise my discussion in the same issue. If he had sent me a copy of his comment, I would have gladly replied so that my response could have been printed in the number showing the illustrations. Comment where the illustrations would not be available would be useless. On that account, it seems desirable here to answer Ottolengui's criticisms, in the description of my illustrations. (See p. 341.) Let not the members of the "100per cent club" jump to the conclusion that, because we differ in some respects, we are engaged in a deadly feud. We are in thorough harmony on the important fact that a certain percentage of pulpless teeth can be maintained in the jaw when properly treated, without becoming a menace to the individual's health. Personally I feel, with Ottolengui, that these differences of opinion should be freely aired, because they involve very vital principles in certain classes of cases. We are all liable to error. If $I$ am in error, I trust I may live long enough to admit it. I believe the correctness of my view will be recognized by the dentists of the future, who will be as thoroughly educated in every branch of medicine as every other specialist in the healing art is supposed to be.

While Ottolengui at heart has no fault to find with my treatment of infected teeth, he and many of my friends are shocked at my utter disregard for the maintenance of what they call the "physiologic encapsulation" in recently devitalized teeth. It is certainly true that many teeth made pulpless by trauma of some kind, and many teeth with imperfect root fillings, have been under years of observation without showing a trace of infection. This condition of affairs is freely acknowledged even by indifferent observers. For this very reason, the lack of a perfect operation does not necessarily spell toxemia, and many able men fail to discover the flaws in their technic.

VII. THE IMPORTANCE OF ENCAPSULATION OF ALL FORAMINAL OPENINGS

At the beginning of this paper I referred to the unsatisfactory experiment, in the ' 80 s by Atkinson and myself, in pulp amputation. 
We left a large amount of pulp tissue in some cases and very little in others; and, though working under aseptic auspices, Atkinson became convinced that the procedure was wrong. William $\mathrm{H}$. Atkinson was the greatest dentist I ever met. Having once come to the conclusion that all of the pulp tissue must be removed, he at once acted accordingly. With a large surgical drill he would go straight down the canal (when feasible) and straight through the foramen, never stopping until the blood flowed into the canal. Then followed intense medication of the periapical region, with the most powerful escharotics to take the place of the knife. He was the first to show me the use of gold for root-canal fillings; but, at the time, he said: "we must find an ideal root filling and it cannot be a cement." Those were the days of the dental giant. I will never forget the first clinic in New York by the beloved McKellops, of St. Louis, in which he filled a root canal with gutta-percha (and used chloroform). When it was over Atkinson exclaimed, "eureka," and gave me such a hug that my ribs were sore for days. Those were the days when oxychloride-of-zinc cement was used for root-canal fillings. We fought the cement root-canal filling at every point because we knew it was wrong.

In voicing his opposition to deliberate insulation of the end of a root, Ottolengui asks: "is there any analogous treatment in the entire realm of surgery?" Teeth do not normally grow in any other place than the alveolar process. Into this process come microörganisms hunting for sustenance, which is most abundant at the end of the root. How much of the alveolar tissue is infected by such microörganisms no one can tell. It is a common error to fail to realize the great extent of the infected region. It is usually forgotten that when a few million germs in the front ranks are destroyed, other millions at once step forward to take their places. These germs are looking for sustenance, which can always be found around any foraminal opening. It is this use, by pathogenic germs, of the tooth as a storehouse for food that makes an infected tooth a menace to health. It is impossible to determine that no more germs will visit this region, but, obtaining no food there, they die rapidly, and are harmless.

The main body of a pulpless root is united by the periodontal membrane to the periosteum of the jaw. Only the root tip is without a 
vital connection. This tip may be just the foraminal canal or it may be a considerable portion of the root end. It is composed of inorganic and organic materials, all capable of retrograde metamorphosis, if it comes in contact with vital tissues. Here is where the wonderful value of insulating this most vulnerable part of the tooth comes in. Base-plate gutta-percha, an inert substance and nonirritating, is utilized to encapsulate this exposed root end. It is true that the encapsulation must be perfect in order to leave the tooth ideally defended. After hundreds of cases have proven the correctness of this therapy, it remains no longer a theory, but a fact. Skepticism and ignorant criticism, however powerfully expressed, cannot remove the roentgenographic record of successes when encapsulation was successfully accomplished. Even the record of observed failures, through inability to make encapsulation a success, becomes a proof of the soundness of this therapy.

If this point is well taken, then it should require little argument to convert dentists to the use of the encapsulation therapy for every pulpless tooth. If no infection has ever been present, the more the need for encapsulation as a great prophylactic measure. It is here that the greatest objection arises to the use of rosin as advocated by Ottolengui. While base-plate gutta-percha is an inert substance, rosin has been found to be filled with organisms and consequently is absolutely unfitted for insulation purposes. In the technic advocated by Ottolengui, the rosin solution under ordinary circumstances must go through the foramen, if the filling is to go anywhere near the end of the foramen. It is easy to see that this material will not do for insulation purposes.

In his criticism of my discussion, Ottolengui makes a great point of showing the air spaces where chloro-percha is dropped on a slab and allowed to harden. No one contradicts the truth of this; in fact we might add that these "igloos" are twice as large when, according to Callahan, rosin is added to the solution. Ottolengui realizes that I shall vigorously answer this statement by saying that we do not fill root canals with chloro-percha, and he attempts to answer my answer before he has heard it. We get rid of these air spaces by long continuous "mortarization" of our gutta-percha. The canal is filled with chloroform into which we introduce our gutta-percha points. The 
canal is the mortar; the plugger is the pestle, which turns around and around the macerated gutta-percha, making pressure uniform against every possible space that the gutta-percha enters. This "mortarization," kept up long enough, gives us a homogeneous mass in every canal space and so compresses the gutta-percha that all spaces disappear. The fact that these root fillings inhibit the passage of any colored fluid should be a sufficient reply to every criticism in this relation. In fact, at the close of Ottolengui's criticism of encapsulation we are met by this paradoxical sentence: "I have little doubt that a homogeneous gutta-percha root filling, inserted with Rhein's technique, will inhibit the entrance of bacteria." As this is the goal of all our root-canal therapy, why the objection?

Unfortunately, many men fail to obtain a true picture of the technique of my operation from reading my articles. It is impossible for me to express in words the delicacy of manipulation, the length of time, and the unflagging patience required of the operator. Only a practical training at the side of the patient, or at the technique table, can make this properly understood. Consequently, I beg my colleagues not to condemn my operation before they have personally seen the operation properly performed.

In the lantern slides I am about to present, I show all kinds of cases-successes and failures-those of real instructive benefit. It shall be my endeavor to reply to Ottolengui's criticisms of them in the appended descriptions.

VIII. DISCUSSION OF THE ILLUSTRATIONS (figs. 1-24); AND FURTHER COMMENT ON CRITICISMS BY OTTOLENGUI

Fig. 1. Shows a gutta-percha root filling, inserted nearly thirtytwo years ago in the upper canine of a young girl, the pulp having been traumatically devitalized. A few years ago the patient, now a mother of quite a family, presented with a traumatic devitalization of the adjoining first bicuspid. The roentgenogram shows a large radiolucent periapical area. Nevertheless, there is no gainsaying the absolute normality, histologically, of the alveolus in which the point of the gutta-percha cone used in the root filling of the canine is protruding. This root filling was not affected by a very bad automobile accident. 
Ottolengui asks: "What does this prove?" It proves conclusively that, notwithstanding the protrusion of the cone of gutta-percha, if everything was aseptic and if the gutta-percha point hermetically sealed the foramen in passing through it, the result must be a return to physiologic tissue. Ottolengui asks: "If the root filling had stopped exactly at the foramen margin, would not the result have been as good?" The answer depends entirely on what Ottolengui means by "foramen margin." If the filling failed, by any unmeasurable length, to seal the outer margin of the foramen, it would not only not have been as good, but it would have been a total failure in preventing bacteria from obtaining sustenance in the crater-like beginning of every foramen. The filling must cover the outer lips of this crater-like opening in order to prevent the entrance of germs.

Figs. 2 and 3. Practically the same kind of case as that in fig. 1 is shown. Although the adjoining first bicuspid was extracted because of an incurable infection, it failed to interfere with the ideal regeneration of the alveolus under the second lower bicuspid.

Fig. 4. A roentgenogram of an upper pulpless lateral showing the diagnostic wire protruding. (See fig. 5.)

Fig. 5. A roentgenogram of the tooth shown in fig. 4, after the root canal had been filled. This is a remarkably interesting and instructive case, and yet Ottolengui failed entirely to appreciate why it had been introduced. In his reply, he claims to match it with a root filling in a lower tooth which apparently shows encapsulation. While the encapsulation in fig. 5 is exceptionally good, it was not introduced to show that fact. Fig. 5 was introduced to show that there was no unusual amount of reaming. The filling, however, shows all kinds of accessory openings leading to the pericementum. Two of these canals are very large in diameter; others not so large. The picture is given in order to demonstrate that while we have an ideal encapsulation of the end, the gutta-percha did not pass through this foramen beyond the encapsulation. It did, however, ideally fill all these subsidiary canals and encapsulate them at the same time. The picture is intended to show how "mortarization" forces the macerated gutta-percha into every opening, of which there were many.

Figs. 6 and 7. These roentgenograms show a lower-bicuspid root canal during the process of root filling. The end of the root shows 


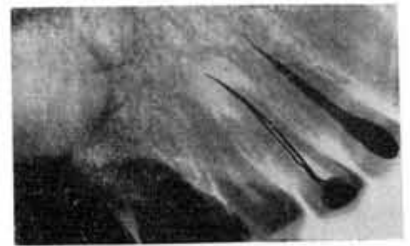

Fig. 1. Roentgenogram of a Protruding Gutta-percha Root Filling; Inserted Nearly Thirty-two Years Ago in an Upper Canine of a Young Girl, the Pulp having been Traumatically Devitalized

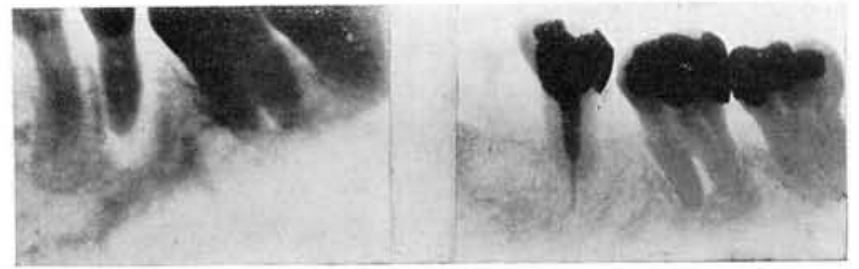

FIG. 2

FIG. 3

Figs. 2 and 3. Roentgenograms of a Case Similar to that Shown in Fig. 1

The figure at the left (2) shows a granuloma at the end of a lower second bicuspid; November 4, 1911. The figure at the right (3) shows ideal regeneration, nine years and three months later; February 11, 1922.

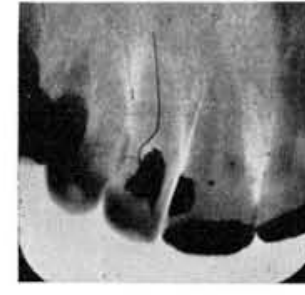

FIG. 4

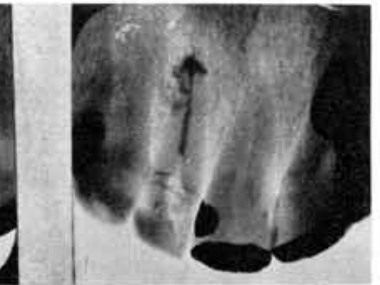

FIG. 5

Figs. 4 and 5. Ideal Filling of the Subsmiary Canals and Perfect Encafsulation without Unnecessary Protrusion

The figure at the left (4) shows an upper pulpless lateral with a diagnostic wire protruding. The figure at the right (5) shows the condition of the tooth after the root canal had been filled.

THE JOURNAL OF DENTAL RESEARCH, VOL. IV, NO. 3 
that an absorption process had been started, exposing some of the cementum, bringing the case under a classification where the percentage of successes is very low because of the necessity of obtaining absolute encapsulation over all exposed cementum. The first picture is taken with a rubber dam in position, and shows what may be termed a filling to the end of the canal. According to Ottolengui, I should have stopped there and considered the operation ideal, even though exposed cementum is shown. On the contrary, "mortarization" of the gutta-percha was continued until (fig. 7) I obtained ideal encapsulation not only of the foramen, but also of the end of the root, including the exposed cementum. It is difficult to understand why Ottolengui should fail to concede that an ideal result was produced in this typical case.

Figs. 8 and 9. These roentgenograms show a similar value of the "mortarization" technic, carried to its ultimate conclusion. Fig. 8 shows a lower molar where an unusual amount of gutta-percha was packed into the mesial canals. With a feeling of confidence the rubber dam was removed, but our disappointment was great to find, from our roentgenogram, that gutta-percha was quite short of the end of the root. Two days later the gutta-percha was re-softened with chloroform and "mortarization" kept up until the beautiful encapsulation shown in fig. 9 was obtained. Note that this time the picture was taken before the rubber dam had been removed.

Fig. 10. The first of these five roentgenograms of an upper bicuspid was taken in January, 1912; the last, in San Francisco, on August 10,1922 . The case has always been the most instructive in my case books, because it clearly proves that (a) the etiologic factors in a case of acute arthritis were the toxins from a traumatically devitalized pulp; that (b) removal of the pulp and insertion of the root filling cured the arthritis; and that (c) there was a steady improvement in the histological character of the regenerated alveolus. The story is well worth recording. Miss K., age 18 (my patient for many years, with very few fillings and an ideally sanitary mouth), in a storm while crossing the ocean to Italy, was thrown from her berth, and her head badly bruised and face disfigured. There was no suspicion of any injury to her teeth. Two weeks after her arrival in Rome a knee commenced to swell, and she was compelled to use crutches for the 


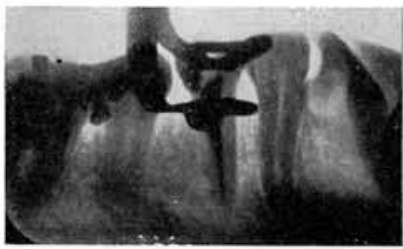

Fig. 6

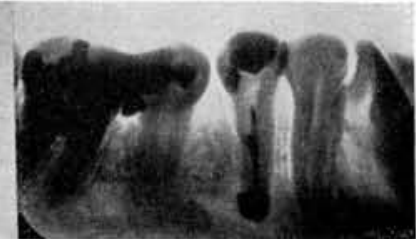

FIG. 7

Figs. 6 and 7. Ideal Encapsulation, after "Mortarization"

The figure at the left (6) shows a lower bicuspid root-canal during the process of root filling; apical absorption had been under way and cementum was exposed. The figure to the right (7) shows ideal encapsulation, not only of the foramen but also at the end of the root, after "mortarization."

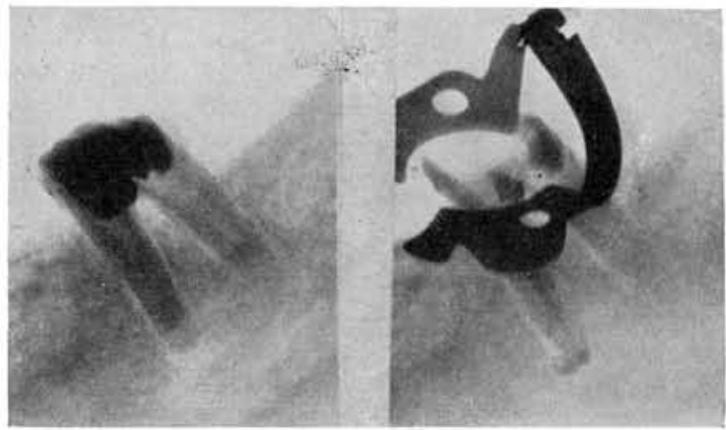

FIG. 8

Fig. 9

Figs. 8 and 9. Perfect Encapsulation, after Re-treatment

The figure at the left (8) shows a lower molar with an unusual amount of gutta-percha packed into the mesial canals. The figure at the right (9) shows beautiful encapsulation. after re-treatment, including softening of the gutta-percha and "mortarization" of it.
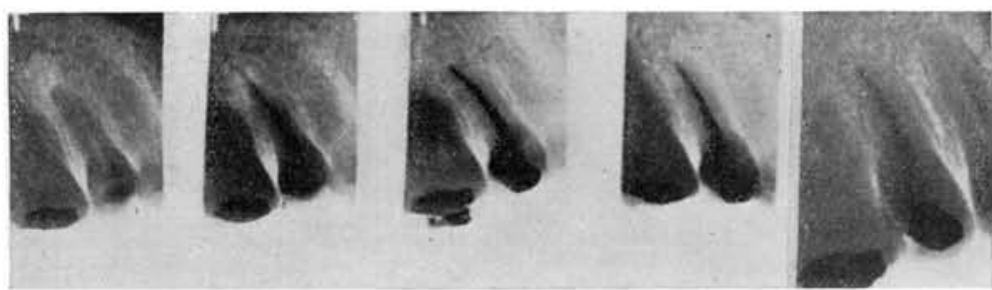

Fig. 10. Steady Improvement in the Character of the Regenerated Alveolus

Roentgenograms taken successively (left to right) in January, 1912; May, 1912; February, 1919; December, 1920; August, 1922. 
year and three-quarters that she remained in Italy. After her return to the United States, she was under the care of a skilled orthopedist, but he was unable to benefit her. About six months after her return, she felt for a few moments a slight irritation over the root of the second bicuspid. A roentgenogram gave us the first indication of the presence of a well marked granuloma. The tooth had no filling in it and was without any defect. Under strict asepsis the pulp chamber was entered and found to be filled with only a brownish fluid. This was siphoned into an aseptic tube and cultured. No growth could be detected before the ninth day. The organism was a typical streptococcus viridans. Zinc electrolytic treatment was given and the root canal filled. The patient was able to discard the crutches after the first week, and up to date there has been no recurrence of arthritis. The pictures show the gradual disappearance of the granuloma and complete osseous regeneration.

In the second picture, in fig. 10, there is a slight excess of protrusion of gutta-percha. As stated previously, there is always a possibility that such a protrusion will break off; it did so in this case. This is not an unusual occurrence, and invariably the small particles of gutta-percha are encysted in the regenerated alveolar structure. This small particle of gutta-percha can be found slightly to the right of the tip of the root. The reproduction of these pictures (first four), in Dental Items of Interest, was so poor that this particle can scarcely be found in them, but even then it can be detected. Ottolengui very disinterestedly hints that the particle might have been exfoliated through a fistula. There has never been a fistulous opening in this case, nor the slightest local disturbance. It might as well be said, here and now, that Ottolengui has only a theoretical understanding of my technic. He has never had any practical instruction in my method. When he assumes the possibility of a secondary infection, he shows a blissful ignorance of how we operate, and illustrates in a practical way the truth of my unhappy conclusion that it is well nigh impossible for any one to appreciate my technic who does not watch it in practice.

I have treated many cases of infection that were so bad that I found them incurable. I have a file of all the original records of my cases dating back to May, 1881. It is impossible to find a case 
in my records, handled by myself, where, after a root canal has been filled, any infection ever started that would naturally result in a fistulous opening. My claim is that, with a proper understanding and execution of aseptic procedure, such a result (very common, I am given to understand) would be impossible. This is no idle boast; and any visiting committee can have the opportunity, at any time, of checking up this statement.

Fig. 11. These roentgenograms show one of the most beautiful cases of regenerated alveolar structure on record. A girl, age 17, had the two lower incisors traumatically devitalized in a basketball game. Treated at first by her dentist, in Halifax, who found himself very much perplexed, especially as a large mass of gutta-percha had been forced through the foramen, she was sent to me on November 21, 1919, when we took the first picture. The second picture was sent to me from Halifax by her dentist, and I could not control the angle at which it was taken. The lump of gutta-percha is just discernible at the bottom of the first picture. This difference in the angle of the picture taken by me, and the one taken by her dentist after regeneration was complete, has again prompted Ottolengui to question the accuracy of my statement that all the bone between the two incisors was missing when she came to me. My statement would not have been so positive if there had been any uncertainty; and the history of the treatment, studied with the aid of the accompanying roentgenograms (figs. 12, 13,14 and 15), is most positive proof of the correctness of my statement.

Figs. 12, 13 and 14. These nine roentgenograms were taken successively on November 21 and December 10, 1919; January 7, 12, 17, 22 and 28, February 3, and March 10, 1920. The very first picture that was taken (fig. 11) showed the dislodged mass of guttapercha in the alveolar space far below the ends of the roots. On the same day, after treatment of one of the teeth, the lump of gutta-percha is shown (in the first picture in fig. 12) to be between the roots, halfway to the crown. All of the succeeding pictures show it in different positions, which means that there was a large empty space between the roots and below them. Electrolytic medication in an intensive manner was administered on the six dates specified for figs. 12 and 13; but, up to January 22, 1920, it was impossible to remove the floating piece of gutta-percha. 


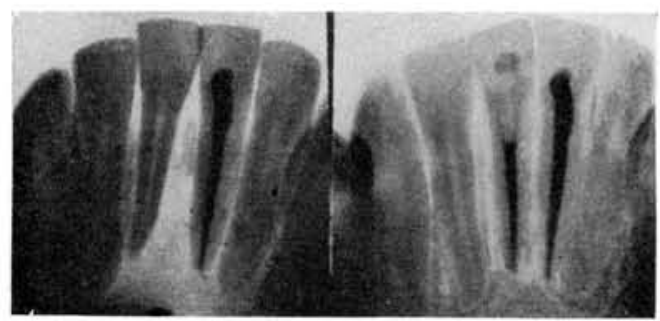

Fig. 11

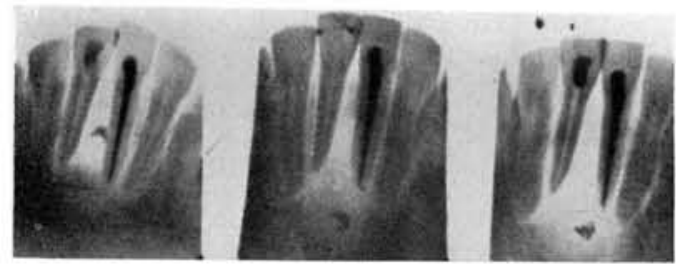

FIG. 12

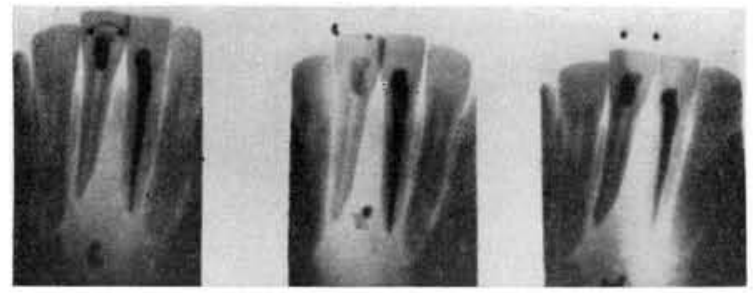

Frg. 13

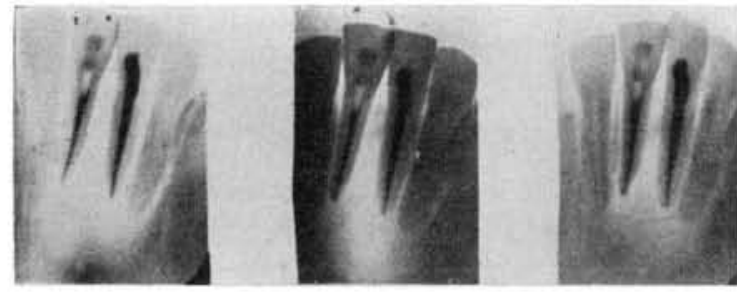

Fig. 14

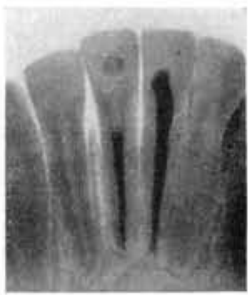

Fig. 15

Figs. 11, 12, 13, 14 and 15. A Case Involving Traumatic Death of Pulps; Imperfect Root Therapy; a Mass of Gutta-percha in the Periapical Region under Treatment; Removal of the Latter; Complete Regeneration

Fig. 11. Shows complete regeneration of alveolar structure. The roentgenogram at the left was taken in November, 1919; that on the right, in January, 1921. See fig. 15.

Fig. 12. Therapy and efforts to remove the particle of gutta-percha continued. The roentgenograms were taken (left to right) on November 21 and December 10, 1919 and on January $7,1920$.

Fig. 13. Therapy and efforts to remove the particle of gutta-percha continued. The roentgenograms were taken (left to right) on January 12, 17 and 22, 1920.

Fig. 14. Therapy continued and the particle of gutta-percha removed. The roentgenograms were taken (left to right) on January 28, February 3, and March 10, 1920.

Fig. 15. Same as the right half of fig. 11; taken in January, 1921. 
On January 28, 1920, the bacteriologic condition had improved to such an extent that we completed both root fillings, made a very large incision between the incisors, and removed the floating piece of gutta-percha. In fig. 14, you will note (February 3) the results of a rapid regenerative process then already in progress. On March 10, this regeneration had advanced much farther. The patient then returned to Halifax.

Fig. 15. This roentgenogram was sent to me from Halifax the following January (1921). The remarkably rapid physiologic regeneration was largely due to the youth of the patient, after all infection had been removed. At the same time, I am convinced that the intensive electrolytic treatment exercised a marked beneficial action on this ideal return to normality.

Some of our cases do not turn out successfully. I shall show some of the failures, in order that it may be seen that we do not claim 100per cent success; and to show, also, that we learn what we can from our failures.

Fig. 16. These roentgenograms were taken from a young woman who was a patient in one of our classes. On December 6, 1918, we started operations. The infected region is well defined. At the next meeting of the class, in January, 1919, the root canal was filled, apparently very successfully. The patient had the root crowned by another dentist. The following December (1919) as the third picture shows, there was marked reinfection. Personally I place the blame for this failure on insufficiency in the amount of applied electrolytic medication. The class wanted to see the root filled, and this was done before a favorable bacteriologic condition was obtained. The lesson taught by this failure was impressive.

Fig. 17. A successful result is shown. (See fig. 18.)

Fig. 18. These roentgenograms show the bicuspid on the opposite side of the mouth of the patient involved in fig. 17. Here again we have a marked failure due, very likely, to a desire to fill the root canal before our bacteriologic condition had sufficiently improved. Both these teeth were extracted. Cases like these have convinced me that, if electrolytic treatment is continued far enough, its possibilities are unlimited.

Fig. 19. This roentgenogram was taken from a woman, aged over 50 , who had been a severe sufferer from arthritis; and also had been 


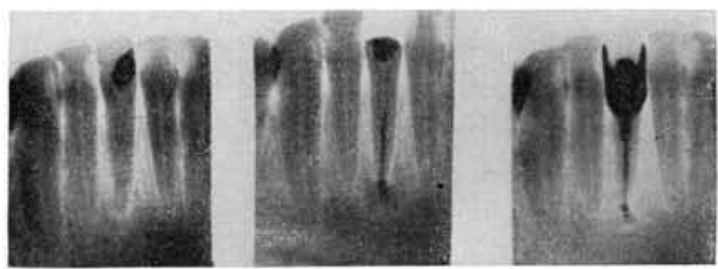

Fig. 16. A FaILURE; ReINFECTION

The roentgenograms were taken (left to right) in December, 1918; January, 1919; and December, 1920.

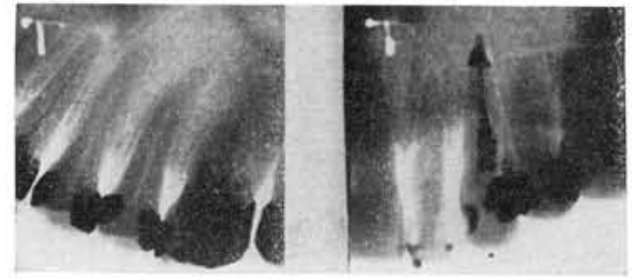

Fig. 17. A Success

The roentgenograms were taken (left) on February 20, 1919, and (right) June 19, 1919.

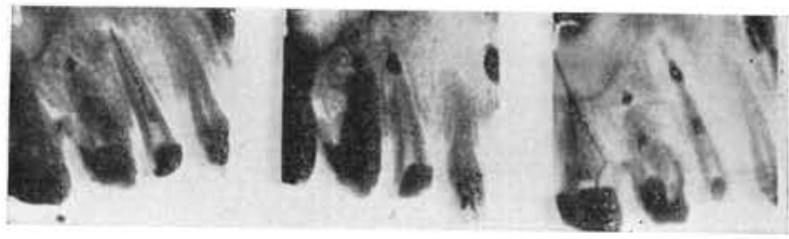

Fig. 18. A FaILltre

The roentgenograms were taken (left to right) on January 24 and 31, and April 4, 1919.

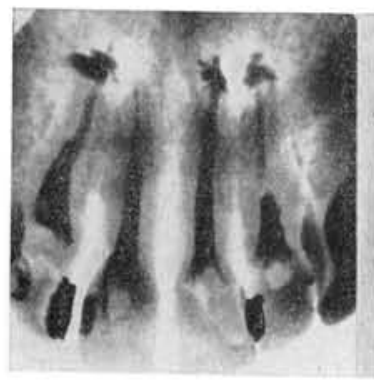

Fig. 19

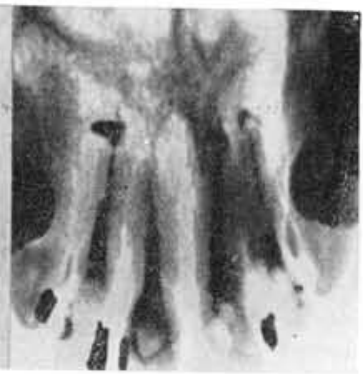

Fig. 20

Figs. 19 AND 20. A SUCCESS

The roentgenograms were taken (19) in 1917 and (20) 1919. 
suffering from tic in the right incisor fossa. The right lateral, left central, and lateral, were pulpless; roentgenograms showed large radiolucent areas. The right central contained a living pulp that was partially calcified and causing continuous pain. This living pulp, as well as the defective root fillings in the other three teeth, were removed. Fig. 19 shows the result immediately after the operations. The patient resided in Georgia and I referred her to Dr. Hinman, of Atlanta, as she required other treatment. She arrived in Atlanta at the time of the annual dental meeting there, in 1917, and was inspected by Drs. Hartzell and Grieves who happened to be there. They were insistent on curing this case at their clinic by amputating the root ends of all four teeth. Dr. Hinman's associate, Dr. Johnston, however, recognized the success of my operation; and the patient, following his advice, refused to be operated upon.

Fig. 20 shows the conditions two years later, ideal regeneration having taken place. Incidentally, every symptom of tic and arthritis disappeared. She has thrown away her cane and is an indefatigable pedestrian. This case teaches us the necessity of being careful not to make a hasty diagnosis. How many of us require this lesson will be left for posterity to determine.

I am reserving my best case for the last. It has been stated by many authorities that where absorption of the root has taken place, so that cementum is exposed, it is useless to endeavor to treat such a case. (See figs. 21-24.)

Fig. 21 shows a lower bicuspid. It must have been pulpless for years, without giving trouble or causing any suspicion of its condition, when, during an intra-oral inspection, this condition revealed itself. The prognosis was so unfavorable that I advised extraction. I was asked to see what could be done, however, and at once determined to use electrolysis "to the limit." The granuloma at that time was well marked. After sufficient treatment had been given, the one hope was to have the gutta-percha thoroughly encapsulate the absorbed root end. How thoroughly we succeeded is revealed in fig. 21, which shows on the left side the granuloma, etc., and on the right side, over a year later, the thoroughly encapsulated root end, with ideal alveolar regeneration. I am convinced that this wonderrul result would never have been attained if it had not been for the long 
continued use of the galvanic current. From February 12 to April 30, 1920, I applied over 25 milliampere hours of current, with iodine solution. It was only after repeated bacteriologic tests had failed to yield growths that, on April 30, 1920, the root canal was effectively filled. A few of the many roentgenograms taken during this treatment are shown in figs. 22-24.

Fig. 22. A roentgenogram taken on March 13, 1920, after six different treatments; shows very little change. The cured condition is seen in the second roentgenogram, taken, at the same angle, on November 23, 1920.

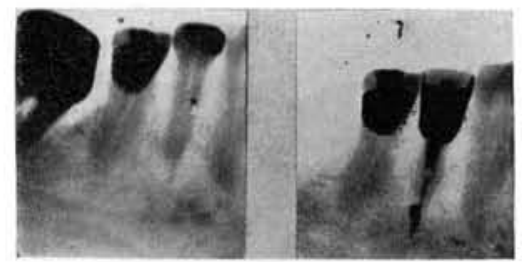

Fig. 21

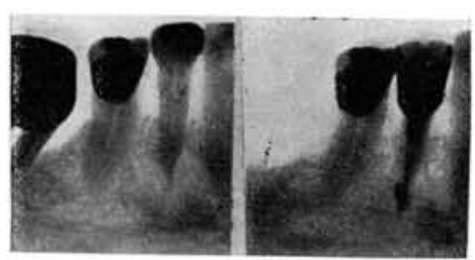

FIg. 22

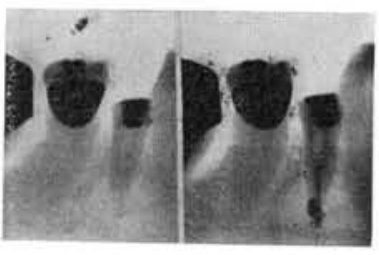

Frg. 23

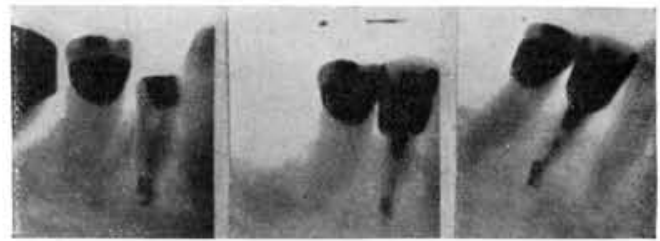

FIG. 24

Figs. 21, 22, 23 and 24. A Success, Showing Complete Regeneration after Perfect Encapsulation

Fig. 21. The roentgenograms were taken (left) on February 12, 1920, and (right) March 3, 1921. The latter shows complete regeneration. See fig. 24.

Fig. 22. The roentgenograms were taken (left) on March 13, 1920 (after six separate electrolytic treatments during a period of a month), and (right) November 23, 1920. Complete regeneration is indicated.

Fig. 23. The roentgenograms were taken (left) on April 23, 1920, and (right) April 30, 1920. The former was taken after the first negative bacteriological test had been obtained; electrolytic medication with iodine was continued. The latter was taken after the second negative bacteriological test had been obtained and the root filled.

Fig. 24. The roentgenograms were taken (left to right) on May 18, and November 11 and 23, 1920. Continuous regeneration is indicated. See fig. 21 . 
Fig. 23. We see the condition on April 23, 1920, when, for the first time, no bacterial growth was obtained. Nevertheless, iodine electrolysis was continued. On April 30, 1920, no growth having developed, zinc electrolysis was used, and the root filled.

Fig. 24 shows the stages of regenerative progress from May to November, 1920. So far as these pictures can be properly reproduced, they tell their own story.

\section{SUMMARY OF GENERAL VIEWS}

When pulpless teeth are retained in the jaw, they should be placed in such a condition that they can offer no sustenance to microörganisms that may reach them. To accomplish this purpose (a) all pulp tissue must be removed from the canal; (b) all pathogenic conditions of the periapical region must be eliminated; and (c) the entrance of every foramen must be hermetically encapsulated so that there will be no possibility of ingress to the interior of the root. The encapsulation becomes an insulating defensive armament against possible infection.

38 E. 61st Street 
